\title{
ON THE CONSTRUCTION OF RING EXTENSIONS
}

\author{
by EDWARD L. GREEN and IDUN REITEN
}

(Received 25 September, 1974)

Let $\Lambda$ denote a basic artin ring and $r$ its radical. In most of this paper we assume that $r^{2}=0$ and that $\Lambda$ is a trivial extension $\Lambda / r \ltimes r$ (see Section 1 for definition). Let $P_{1}, \ldots, P_{n}$ be the non-isomorphic indecomposable projective (left) $\Lambda$-modules, and consider triples $\left(P_{i}, M_{i}, u_{i}\right)$, where the $M_{i}$ are (left) $\Lambda$-modules and $u_{i}: r P_{i} \rightarrow M_{i} / r M_{i}$ isomorphisms. From this data we construct a new ring $\Gamma$, which in "nice cases" has the property that $r^{\prime 3}=0$, $\Gamma / r^{\prime 2} \cong \Lambda$, and $r^{\prime} Q_{i} \cong M_{i}$ as (left) $\Lambda$-modules, where the $Q_{i}$ are the indecomposable projective (left) $\Gamma$-modules and $r^{\prime}$ is the radical of $\Gamma$.

Especially interesting are the cases where $\Gamma$ is selfinjective, of which we give a complete description. When in this case $r^{\prime 2}=\operatorname{soc} \Gamma$, then, since the indecomposable summands of $\Gamma$ are the only indecomposable $\Gamma$-modules which are not annihilated by soc $\Gamma$, the representation theories for $\Lambda$ and $\Gamma$ are closely related. In particular, our results put into a general context an example of a particular selfinjective ring given in [7], and should be useful for constructing other examples of selfinjective rings with certain given properties.

Another special choice for the $M_{i}$ we consider is for all of them to be projective. This turns out to give a way of writing $\Lambda$ with $r^{2}=0$ and gl.dim. $\Lambda=n>1$ as a factor ring of a ring $\Gamma$ with $r^{3}=0$ and lower global dimension. We provide a formula for gl.dim. $\Gamma$ in terms of $n$.

We hope that other particular choices for the $M_{i}$ (or even for the $P_{i}$ ) will give other interesting ring constructions, for the case $r^{2}=0$, and also by making appropriate generalizations to the case $r^{2} \neq 0$.

As for $r^{2} \neq 0$, we start in Section 5 a discussion of when, for an artin ring $\Lambda$ of Loewy length $n \geqq 2$, written $L L(\Lambda)=n$ (i.e. $r^{n}=(0), r^{n-1} \neq(0)$ ), there is a selfinjective ring $\Gamma$, with $L L(\Gamma)=n+1$, and $\Gamma / r^{\prime n} \cong \Lambda$. For this to be the case it is necessary that there is a pairing of the indecomposable projective and injective $\Lambda$-modules satisfying certain conditions (see Theorem 5.1). Our discussion in this section enables us to obtain more complete results also for artin rings $\Lambda$ of Loewy length 2 .

All our modules will be finitely generated left modules unless otherwise stated, and the category of finitely generated left $\Lambda$-modules will be denoted by $\bmod \Lambda$.

We are grateful to Professor Maurice Auslander for inspiring us to doing this work.

1. Let $\Lambda$ be an artin ring. Assume that the square of the radical $r$ is zero, and that $\Lambda=\Lambda / r \ltimes r$, i.e. that the elements are pairs $(a, b)$ with $a \in \Lambda / r, b \in r$, addition is componentwise, and multiplication is given by $(a, b) \cdot\left(a^{\prime}, b^{\prime}\right)=\left(a a^{\prime}, a b^{\prime}+b a^{\prime}\right)$. Assume also for the rest of the paper that $\Lambda$ is basic, i.e. that $\Lambda / r$ is a product of division rings. Let $P_{1}, \ldots, P_{n}$ be the indecomposable non-isomorphic (left) projective $\Lambda$-modules, and consider triples $\left(P_{i}, M_{i}, u_{i}\right)$, where $M_{i}$ is a $\Lambda$-module and $u_{i}: r P_{i} \rightarrow M_{i} / r M_{i}$ an isomorphism. On the basis of these triples, we shall construct a new artin ring $\Gamma$, which under certain conditions will have $\Lambda$ as a factor ring in a natural way, and for which $r^{\prime 3}=0$. 
A quick description of the $\Gamma$ we construct can be given as follows. Let $M=\coprod_{i=1}^{n} M_{i}$, and $u: r \rightarrow M / r M$ be the isomorphism induced from the $u_{i}$. We then consider the graded module $\Lambda / r+r+r M$ over the graded ring $\Lambda / r+r+r \otimes r$, where the map $r \otimes r \rightarrow r M$ is given by $r \otimes r \stackrel{1 \otimes u}{\rightarrow} r \otimes M / r M \rightarrow r M$ where the last map is the natural one. We define $\Gamma$ to be the opposite of the endomorphism ring of this graded module over the graded ring $\Lambda / r+r+r \otimes r$.

It will, however, be useful to use a more explicit description of the elements of $\Gamma$. We precede this by giving a description of the $\Lambda$-modules, which is closely related to and easily deduced from the description of modules over trivial extensions given in [5]. As a $\Lambda / r$-module, a $\Lambda$-module $A$ is the direct sum $A=A / r A \amalg r A$. A map between two $\Lambda$-modules $A$ and $B$ is then a triple $(a, b, c)$, where $a: A / r A \rightarrow B / r B, b: r A \rightarrow r B$ and $c: A / r A \rightarrow r B$ are such that

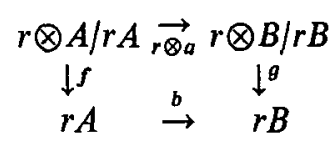

commutes, where $f$ and $g$ are the natural maps. If $a: A / r A \rightarrow B / r B$ is a given map, there is at most one choice for $b$ since $f$ is an epimorphism. Hence a map is determined by a pair $(a, c)$, where $a: A / r A \rightarrow B / r B$ cannot be arbitrary, but restricted by the requirement that it is possible to find some $b: r A \rightarrow r B$ such that the above diagram commutes. $c: A / r A \rightarrow r B$ is, however, arbitrary.

We write the graded module $\Lambda / r+r+r M$ as $(\Lambda, M)$, and similarly $P / r P+r P+r N$ as $(P, N)$, for a direct summand of a sum of copies of $(\Lambda, M)$. A map from $(P, N)$ to $\left(P^{\prime}, N^{\prime}\right)$ is then a triple $(f, g, h)$, where $f: P \rightarrow P^{\prime}, g: N \rightarrow N^{\prime}, h: P \rightarrow N^{\prime}$, with some additional conditions on the connection between the maps $f, g$ and $h$. By what we have seen above, each of these maps can be described as a triple, and in doing so, the connection is given in our notation as follows:

$$
f=(\alpha, \beta, a), g=(\beta, \gamma, b), h=(a, b, c) .
$$

A map $(f, g, h):(\Lambda, M) \rightarrow(\Lambda, M)$ is really determined by the pair $(\alpha, h), \alpha: \Lambda / r \rightarrow \Lambda / r$, $h: \Lambda \rightarrow M$, where $h$ is arbitrary, and $\alpha$ belongs to a subalgebra $D$ of $\operatorname{End}_{\Lambda}(\Lambda / r)^{\text {op }}$, consisting of the $\alpha$ for which we can find $\beta$ and $\gamma$. Since $\Lambda$ is projective, we can always find $\beta$. Of special interest will be the case $D=\operatorname{End}_{\Lambda}(\Lambda / r)^{\text {op }}$, i.e. the case where $\alpha: \Lambda / r \rightarrow \Lambda / r$ in $(\alpha, h)$ is arbitrary.

We want to study the connection between the rings $\Lambda$ and $\Gamma$. There is a natural map from $\Gamma^{\mathrm{op}}$ to $\Lambda^{\mathrm{op}}$ (hence from $\Gamma$ to $\Lambda$ ) given by sending a map $(f, g, h):(\Lambda, M) \rightarrow(\Lambda, M)$ to $f: \Lambda \rightarrow \Lambda$. This ring map is then a surjection if and only if, given $f$, we can find a triple $(f, g, h)$. By our above remarks, this is the case if and only if the subalgebra $D$ of $\operatorname{End}_{\Lambda}(\Lambda / r)^{\mathrm{op}}$ as defined above is equal to $\operatorname{End}_{\Lambda}(\Lambda / r)^{\text {op }}$.

Assume now that $D=\operatorname{End}_{\Lambda}(\Lambda / r)^{\text {op }}$, and call this condition $A$, so that $\Lambda$ is in a natural way a factor ring of $\Gamma$. It is then easy to see that the kernel $I$ of the natural ring map from $\Gamma$ to $\Lambda$ has as elements the triples $(0,0, h)$, where $h(\Lambda) \subset r M$. We now want to describe the radical $r^{\prime}$ of $\Gamma$. It is then convenient to consider the elements of $\Gamma$ as pairs $(\alpha, h)$, where $\alpha: \Lambda / r \rightarrow \Lambda / r$, $h: \Lambda \rightarrow M$. Clearly the nilpotent elements are the pairs $(0, h)$, i.e. they are given by the maps $h: \Lambda \rightarrow M$, so $r^{\prime}$ is the ideal in $\Gamma$ whose elements are the $(0, h), r^{\prime}$ has a natural structure as a left $\Lambda$-module and is as such clearly isomorphic to $M$. Since $r^{\prime}$ is a two-sided $\Lambda$-module, there is induced a natural two-sided $\Lambda$-module structure on $M$. 
The natural $\Lambda$-isomorphism between $r^{\prime}$ and $M$ induces a natural $\Lambda$-isomorphism between $r^{\prime 2}$ and $r M$, so that $r^{\prime 2}$ consists exactly of the pairs $(0, h)$, where $h(\Lambda) \subset r M$. Hence $I=r^{\prime 2}$, so that $\Gamma / r^{\prime 2} \cong \Lambda$.

Since we are mainly interested in the cases where $\Lambda$ is a factor ring of the $\Gamma$ we construct, we would like to know when our condition $A$ is satisfied. We shall end this section by providing a sufficient condition for this to be the case. Also, for both the special cases we consider later in the paper, $\Lambda$ will be a factor ring of $\Gamma$. We say that $\Lambda$ is a special $k$-algebra (where $k$ is a field) if $\Lambda / r=k \times \ldots \times k$, and the action of $k$ on $\Lambda / r$ is along the diagonal.

Proposition 1.1. Let $\Lambda$ be a special $k$-algebra with $r^{2}=0$, and $\left(P_{i}, M_{i}, u_{i}\right)$ triples with the $P_{i}$ the projective indecomposable $\Lambda$-modules and the $u_{i}: r P_{i} \rightarrow M_{i} / r M_{i}$ isomorphisms. Then $\Lambda$ is in a natural way a factor ring of the $\Gamma$ we construct.

Proof. Consider a fixed triple $(P, M, u)$, where $P$ is indecomposable projective. Let $\alpha$ be a non-zero map from $P / r P$ to $P / r P$, and $\beta=1, \otimes \alpha: r P \rightarrow r P$. Since each such map is clearly given by multiplication by an element in $k$, this is easily seen to be the case also for the induced map $M / r M \rightarrow M / r M$. Hence we can find an associated map $\gamma: r M \rightarrow r M$, so that our condition $A$ holds, and consequently $\Lambda$ is a factor ring of $\Gamma$ in a natural way.

We point out that condition $A$ is not always satisfied for an artin ring $\Lambda$ with $r^{2}=0$, and triples $\left(P_{i}, M_{i}, u_{i}\right)$, not even if $\Lambda$ is a finite dimensional algebra over a field. For let $\Lambda=\left(\begin{array}{cc}K & 0 \\ { }_{k} K_{K} & k\end{array}\right)$, where $K \supset k$ are fields, and $K$ is of dimension 2 as a vector space over $k$. With $P_{1}=\left(\begin{array}{l}K \\ K\end{array}\right), P_{2}=\left(\begin{array}{l}0 \\ K\end{array}\right), M_{1}=\left(\begin{array}{l}K \\ k\end{array}\right), M_{2}=0$, and the $u_{l}$ natural maps, condition $A$ is easily seen not to hold.

2. In this section we investigate when our construction gives a selfinjective ring $\Gamma$ such that $\Gamma / r^{\prime 2} \cong \Lambda$, where $r^{\prime}$ is the radical of $\Gamma$. This case is especially interesting from the point of view of representation theory. For if $\Gamma$ is selfinjective with $r^{\prime 3}=0$ and $r^{\prime 2} Q \neq 0$ for each indecomposable projective $\Gamma$-module $Q$, then $r^{\prime 2}=\operatorname{soc} \Gamma$, and all the indecomposable $\Gamma$-modules except the projective ones are annihilated by soc $\Gamma$, so that the additive categories mod $\Lambda$ and mod $\Gamma$ are closely related. In particular, $\Lambda$ is of finite representation type, i.e. has only a finite number of indecomposable finitely generated modules, if and only if $\Gamma$ is. The necessary and sufficient conditions we find on the triples $\left(P_{i}, M_{i}, u_{i}\right)$ for $\Gamma$ to be selfinjective (such that $\Gamma / r^{\prime 2} \cong \Lambda$ ), put into a general setting an example given in [7] of a selfinjective ring of finite representation type with some special properties, and should be useful for construction of selfinjective rings in other contexts.

We first make the interesting observation that, for our $\Gamma$ to be selfinjective, it is necessary that condition $A$ holds, so that $\Lambda$ becomes in a natural way a factor ring of $\Gamma$. Assume to the contrary that $\Gamma$ is selfinjective, but that condition $A$ does not hold. Then there is an indecomposable projective $\Lambda$-module $P$ and a non-zero map $\alpha: P / r P \rightarrow P / r P$, such that for the triple $(P, N, u)$, we can find no associated $\gamma: r N \rightarrow r N$. It is convenient to look at mod $\Gamma$ as the category of finitely presented functors from the additive category generated by the $\left(P_{i}, M_{i}\right)$ to abelian groups (see [1], Section 4). An indecomposable projective object in mod $\Gamma$ is then a 
representable functor $\left(,\left(P_{i}, M_{i}\right)\right)$. Since each simple $\Gamma$-module is contained in some $\left(,\left(P_{i}, M_{i}\right)\right)$, there is a non-zero map $\left(\alpha^{\prime}, h^{\prime}\right):(P, N) \rightarrow\left(P_{i}, M_{i}\right)$ for some $i$, such that $\operatorname{Im}\left(,\left(\alpha^{\prime}, h^{\prime}\right)\right)$ is simple. Clearly $\alpha^{\prime}=0$, and for $h^{\prime}: P \rightarrow M_{i}, \operatorname{Im} h^{\prime}$ is simple. Consider then the map $\left(0, h^{\prime} \alpha\right):(P, N) \rightarrow\left(P_{i}, M_{i}\right)$. Clearly also $\operatorname{Im}\left(,\left(0, h^{\prime} \alpha\right)\right)$ is simple, but must be different from $\operatorname{Im}\left(,\left(0, h^{\prime}\right)\right)$, by our choice of $\alpha$. This contradicts the fact that $\left(,\left(P_{i}, M_{i}\right)\right)$ has simple socle, and consequently condition $A$ is a consequence of $\Gamma$ being selfinjective.

We now go on to prove the main result of this section. Here we need to assume that $\Lambda$ is an artin algebra, i.e. an artin ring which is a finitely generated module over its centre.

THEOREM 2.1. Let $\Lambda=\Lambda / r \propto r$ be an artin algebra with $r^{2}=0$, and $\left(P_{i}, M_{i}, u_{i}\right)$ triples with $P_{1}, \ldots, P_{n}$ the indecomposable projective $\Lambda$-modules, and the $u_{i}: r P_{i} \rightarrow M_{i} / r M_{i}$ isomorphisms. Then our $\Gamma$ is selfinjective and $r^{2} Q \neq 0$ for each indecomposable projective $\Gamma$-module if and only if the $M_{1}, \ldots, M_{n}$ are the non-isomorphic indecomposable injective $\Lambda$-modules, and condition $A$ is satisfied for the triples. And if $\Gamma$ is selfinjective, then $\Gamma / r^{2} \cong \Lambda$.

Proof. Assume that $\Gamma$ is selfinjective and that $r^{2} Q \neq 0$ for each indecomposable projective $\Gamma$-module. Then we already know that $\Gamma / r^{\prime 2} \cong \Lambda$. Since $\Gamma$ is an injective $\Gamma$-module, we conclude from $\left[3\right.$, p. 30] that $\operatorname{Hom}\left(\Gamma / r^{\prime 2}, \Gamma\right) \cong r^{\prime}$ is an injective $\Lambda$-module. Since $M$ is isomorphic to $r^{\prime}$ as a $\Lambda$-module, it follows that $M$ is an injective $\Lambda$-module. Since the socles of the non-isomorphic indecomposable projective $\Gamma$-modules are non-isomorphic, it follows that the different $M_{l}$ are indecomposable non-isomorphic.

Assume conversely that the $M_{i}$ are the different non-isomorphic indecomposable injective $\Lambda$-modules and that condition $A$ holds. Since $r^{\prime 2} \cong r M$ as $\Lambda$-modules, where $r$ denotes the radical of $\Lambda$, and each $r M_{i}$ is simple, we conclude that the socle of each indecomposable projective $\Gamma$-module is simple. Hence we have a monomorphism $\Gamma \rightarrow E$, where $E$ is the direct sum of one copy of each of the indecomposable injective $\Gamma$-modules. To show that this monomorphism is an isomorphism, consider the usual duality $D: \bmod \Gamma \rightarrow \bmod \Gamma^{\text {op }}$ for artin algebras, and let $R$ denote the centre of $\Gamma$. Since $D(E)=\Gamma^{\text {op }}$, and $D$ preserves length, we have that, since $R$ is commutative, $E$ and $\Gamma$ have the same length as $R$-modules. This completes the proof that $\Gamma$ is selfinjective.

The question remains open if Theorem 2.1 holds when dropping the condition that $\Lambda=\Lambda / r \propto r$ is an artin algebra, and just assuming that $\Lambda=\Lambda / r \propto r$ is an artin ring. We do, however, in Section 5, find another condition on $\Lambda$, which together with our conditions in Theorem 2.1 will be necessary and sufficient in this case.

3. In this section we shall look at another way of writing an $\operatorname{artin} R$-algebra $\Lambda$ as a factor ring of a selfinjective ring, namely the selfinjective ring $\Omega=\Lambda \ltimes E$, where $E=\operatorname{Hom}_{R}(\Lambda, R)$ (see[1], and for a more general construction [5], Section 4). If $r^{2}=0$, where $r$ denotes the radical of $\Lambda$, then $r^{\prime 3}=0$, where $r^{\prime}$ denotes the radical of $\Omega$. Here $\Lambda$ can be an arbitrary artin algebra (with $r^{2}=0$ ), but we do not in general have such a close connection between the additive structures of $\bmod \Lambda$ and $\bmod \Omega$ as we had between $\bmod \Lambda$ and $\bmod \Gamma$ when $\Gamma$ was selfinjective. In general $\Lambda$ can be of finite representation type while $\Omega$ is of infinite representation type. It is, however, possible to investigate when $\Omega$ is of finite representation type, when $\Lambda$ is a special $k$-algebra with $r^{2}=0$, by using results of Gabriel [6], which we shall first recall (see [2, Part II, 
Chapter 1]) for a more detailed treatment). We remark that Gabriel's results have been generalized to arbitrary artin algebras with $r^{2}=0$ by Dlab-Ringel and W. Müller, but we shall still restrict to the case of special $k$-algebras in what follows.

Let $\Lambda$ be a special $k$-algebra with $r^{2}=0, S_{1}, \ldots, S_{n}$ the non-isomorphic simple $\Lambda$-modules and $P_{1}, \ldots, P_{n}$ their projective covers. Then we construct a diagram associated with $\Lambda$ in the following way. Write two rows of points, in each row a point $i$ for each simple module $S_{i}$. For each $P_{i}$, decompose $r P_{i}$ into a direct sum of simple modules $\Sigma S_{j}$. For each $j$ in such a decomposition of $r P_{i}$, draw a line from the point $i$ in the upper row to the point $j$ in the lower row. If $\Lambda$ is given in the form $\left({ }_{1} k_{1} \times \ldots \times{ }_{n} k_{n}\right) \times \Sigma_{j} k_{i}$, where ${ }_{j} k_{i}$ denotes the two-sided $\Lambda / r$ module where the only ${ }_{l} k_{l}$ acting non-trivially on the left is ${ }_{j} k_{j}$ and on the right is ${ }_{i} k_{i}$, and then the action is by multiplication in $k$. Then the diagram is described by two rows of the points $1, \ldots, n$, and a line from $i$ in the upper row to $j$ in the lower row for each ${ }_{j} k_{i}$.

Then by [6] $\Lambda$ is of finite representation type if and only if the associated diagram $K(\Lambda)$ as described above is a disjoint union of diagrams of the following types.

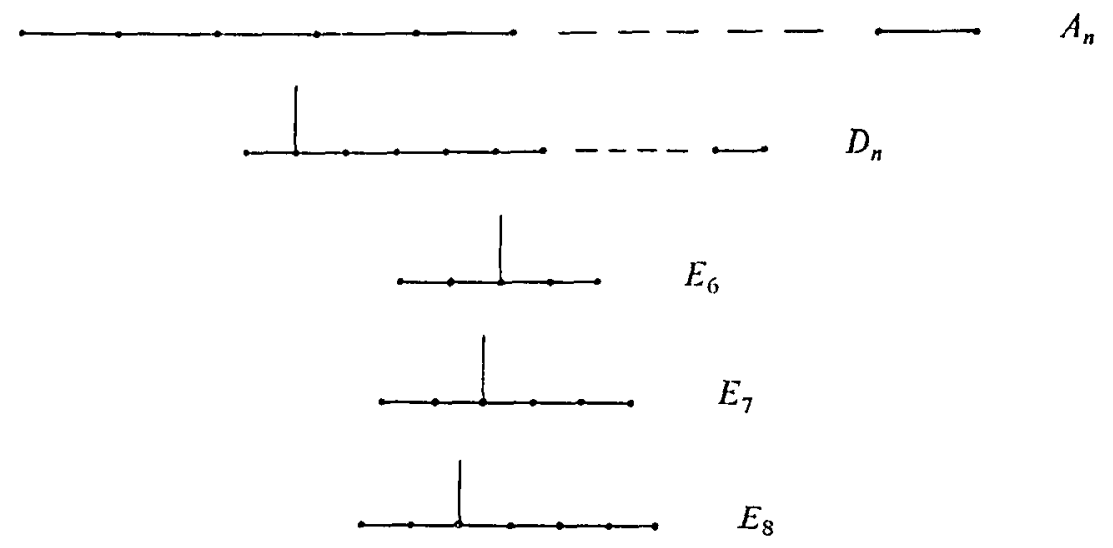

To be able to use this to determine the representation type of $\Omega$, which is the same as the representation type of $\Omega / r^{\prime 2}=\Lambda \ltimes E / r E+E r$, we shall need the following result.

Proposition 3.1. Let $\Lambda=\Lambda / r \times r$ be a special $k$-algebra. Then the diagram for $\Omega / r^{2}$ can be obtained from the diagram for $\Lambda$ by in addition, for each line from $i$ in the top row to $j$ in the bottom row, adding an arrow from $j$ in the top row to $i$ in the bottom row. One must also add an arrow from $i$ in the top row to $i$ in the bottom row if there is no arrow leaving $i$ on the top and no arrow entering $i$ on the bottom.

Proof. Let $E=\operatorname{Hom}_{k}(\Lambda, k)$, and let $\Lambda^{\prime}=\Lambda \otimes_{k} \Lambda^{\mathrm{op}}$. The two-sided $\Lambda$-modules may and will be considered left $\Lambda^{\prime}$-modules. Now $\operatorname{Hom}_{k}\left(\operatorname{soc}_{\Lambda^{\prime}}(\Lambda), k\right)=E / r E+E r$. This can be seen using the fact that for any two-sided $\Lambda$-module $M, \operatorname{Hom}_{k}\left(\operatorname{soc}_{\Lambda^{\prime}} M, k\right)=\operatorname{Hom}_{k}(M, k) / r^{\prime} \operatorname{Hom}_{k}(M, k)$. Here $r^{\prime}$ denotes the radical of $\Lambda^{\prime}$. We also need to observe that $r^{\prime} E=r E+E r$. Since $\operatorname{Hom}_{k}\left({ }_{i} k_{j}, k\right) \cong{ }_{j} k_{i}$ and $\operatorname{soc}_{\Lambda^{\prime}}(\Lambda)=r+\amalg_{i} k_{i}$, where the sum is taken over the $i$ such that ${ }_{i} k_{i}$ is 
both a projective and injective left $\Lambda$-module, the proof is complete, using the description above.

Note that if $\Lambda$ is indecomposable as a ring with $r \neq 0$, then $\Lambda$ has no simple injective projectives and hence the description of $\Omega / r^{\prime 2}$ is somewhat simpler.

This result enables us, for a given special $k$-algebra $\Lambda$ with $r^{2}=0$, to decide whether $\Omega=\Lambda \ltimes E$ is of finite representation type. To illustrate, we give the following general results, which at the same time shows that we can find several examples where $\Lambda$ and $\Omega$ have the same representation type, and several where $\Lambda$ and $\Omega$ do not have the same representation type.

Proposition 3.2. Let $\Lambda$ be a special $k$-algebra with $r^{2}=0$, of finite representation type.

(a) If $\Lambda$ is hereditary, then $\Lambda \times E$ is of finite representation type.

(b) If gl.dim. $\Lambda=\infty$, then $\Omega=\Lambda \ltimes E$ is of infinite representation type.

Proof. (a) It is easy to see that if $\Lambda$ is hereditary, and the associated diagram $K(\Lambda)$ has a line from $i$ in the top row to $j$ in the bottom row, then $j$ in the top row and $i$ in the bottom row are isolated points. Hence if $K(\Lambda)$ is a disjoint union of diagrams of type $A_{n}, D_{n}, E_{6}, E_{7}, E_{8}$, then $K\left(\Omega / r^{\prime 2}\right)$ has the same property.

(b) Let $(i, j)$ denote a line connecting $i$ in the top row with $j$ in the bottom row. If gl.dim. $\Lambda=\infty$, then $\operatorname{pd}_{\Lambda} S=\infty$ for some simple $\Lambda$-module $S$. For the diagram $K(\Lambda)$ this is easily seen to mean that we have lines $\left(i_{1}, i_{2}\right),\left(i_{2}, i_{3}\right), \ldots,\left(i_{n}, i_{1}\right)$. By Proposition 3.1 , the diagram $K\left(\Omega / r^{\prime 2}\right)$ has, in addition, lines $\left(i_{2}, i_{1}\right),\left(i_{3}, i_{2}\right), \ldots,\left(i_{n}, i_{n-1}\right),\left(i_{1}, i_{n}\right)$. Considering $\left(i_{1}, i_{2}\right),\left(i_{3}, i_{2}\right),\left(i_{3}, i_{4}\right),\left(i_{5}, i_{4}\right), \ldots,\left(i_{n-1}, i_{n}\right),\left(i_{1}, i_{n}\right)$, we see that $K\left(\Omega / r^{\prime 2}\right)$ has a cycle, and consequently $\Omega / r^{\prime 2}$, and hence $\Omega$, is of infinite representation type.

4. Let $\Lambda=\Lambda / r \ltimes r$ again be a basic artin ring with $r^{2}=0$, and $P_{1}, \ldots, P_{n}$ the different (non-isomorphic) indecomposable projective $\Lambda$-modules. Let as usual the $\left(P_{i}, M_{i}\right)$ be pairs such that $r P_{i} \cong M_{i} / r M_{i}$, and let $\Gamma$ denote the algebra with $r^{3}=0$ we constructed on the basis of this information. In this section we study this construction for the case in which the $M_{l}$ are projective. It is then easy to see that in this case our condition $A$ is satisfied, so that $\Lambda$ is in a natural way a factor ring of $\Gamma$. In fact, it is easy to see that $\Gamma \cong \Lambda / r+r+r \otimes r$.

We shall here be mainly concerned with the connection between the global dimensions of $\Lambda$ and $\Gamma$. This will give us a way of writing $\Lambda$ as a factor ring of a ring with $r^{3}=0$ of lower global dimension. In [4] it is shown that an artin ring $\Omega$ such that $\Omega / r^{2}$ is a trivial extension, is hereditary if and only if, for some $n, \Omega \cong \Omega / r+r+r \otimes r+\ldots+r \otimes \ldots \otimes r$, and $r \otimes \ldots \otimes r$ $\left(n+1\right.$ times) is zero; and if $\Omega$ is of this type, then gl.dim. $\Omega / r^{2}=n$. In particular, if gl.dim. $\Lambda=2$, then gl.dim. $\Gamma=1$.

On the basis of the above one might be led to conjecture that if gl.dim. $\Lambda=n>1$, then gl.dim. $\Gamma=n-1$. This does not hold for $n>2$. In fact, we have the following result.

THEOREM 4.1. For $n \geqq 1$, we have

(a) $\operatorname{gl} . \operatorname{dim} . \Gamma=2 n \Leftrightarrow \operatorname{gl} . \operatorname{dim} . \Lambda=3 n$;

(b) gl.dim. $\Gamma=2 n+1 \Leftrightarrow$ gl.dim. $\Lambda$ is $3 n+1$ or $3 n+2$. 
Proof. Let $S_{1}$ be a simple $\Lambda$-module (which is then also a simple $\Gamma$-module), and denote by $P_{1}$ its projective cover as a $\Lambda$-module. Let $P_{2}$ be the projective cover of $r P_{1}, P_{3}$ the projective cover of $r P_{2}$, and so forth. It is easy to see that a $\Lambda$-module $M$ is a projective $\Gamma$-module if and only if $M$ and $r M$ are projective $\Lambda$-modules. Consider now the following exact sequences, which give a projective resolution of $S_{1}$ as a $\Lambda$-module and as a $\Gamma$-module respectively.

$$
\begin{aligned}
& 0 \rightarrow r P_{1} \rightarrow P_{1} \rightarrow S_{1} \rightarrow 0 \quad 0 \rightarrow P_{2} \quad \rightarrow\left(P_{1}, P_{2}\right) \quad \rightarrow S_{1} \rightarrow 0 \\
& 0 \rightarrow r P_{2} \rightarrow P_{2} \rightarrow r P_{1} \rightarrow 0 \quad 0 \rightarrow r P_{3} \rightarrow\left(P_{2}, P_{3}\right) \quad \rightarrow P_{2} \quad \rightarrow 0
\end{aligned}
$$

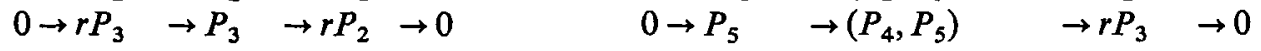

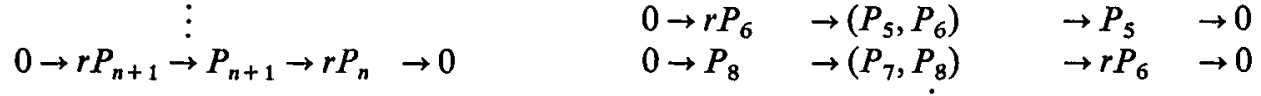

$$
\begin{aligned}
& 0 \rightarrow r P_{3 n} \rightarrow\left(P_{3 n-1}, P_{3 n}\right) \rightarrow P_{3 n-1} \rightarrow 0 \\
& 0 \rightarrow r P_{3 n+2} \rightarrow\left(P_{3 n+1}, P_{3 n+2}\right) \rightarrow r P_{3 n} \rightarrow 0
\end{aligned}
$$

From this we conclude that $\operatorname{pd}_{\Lambda} S_{1} \leqq 3 n$ if and only if $\operatorname{pd}_{\Gamma} S_{1} \leqq 2 n$, and $\operatorname{pd}_{\Lambda} S_{1} \leqq 3 n+2$ if and only if $\mathrm{pd}_{\Gamma} S_{1} \leqq 2 n+1$. As it is well known that the global dimension of an artin ring is the supremum of the projective dimension of the simple modules, the proof is complete.

It would be interesting to know if for a given $\Lambda$ as above, with gl.dim. $\Lambda=n$, it is possible to write $\Lambda$ as a factor ring of an artin ring $\Gamma^{\prime}$ with $r^{3}=0$, where gl.dim. $\Gamma^{\prime}<\operatorname{gl}$.dim. $\Gamma$. Clearly for $n=2$, our $\Gamma$ gives the lowest possible value.

5. Let $\Lambda$ be an artin ring (as usual basic) of Loewy length $n \geqq 2$, denoted by $L L(\Lambda)=n$, i.e. $r^{n}=0, r^{n-1} \neq(0)$. We shall here try to generalize our results from Section 2 , and study the question of the existence of a selfinjective ring $\Gamma$ with $L L(\Gamma)=n+1$, and $\Gamma / r^{\prime \prime} \cong \Lambda$, where $r^{\prime}$ denotes the radical of $\Gamma$. In Section 2 we considered the existence of such a $\Gamma$ for the case in which $L L(\Lambda)=2$ and $\Lambda=\Lambda / r \times r$ is an artin algebra. As a consequence of our discussion in this section, we get some necessary conditions for $\Lambda$ to satisfy, but we do not know if they are sufficient in general. If $L L(\Lambda)=2$ and $\Lambda=\Lambda / r \ltimes r$, we do find necessary and sufficient conditions.

The existence of such a $\Gamma$ is interesting, since also in this more general situation, $\Gamma$ is of finite representation type if and only if $\Lambda$ is.

Before proving the main result of this section, we need to develop one more notion. Let $\Gamma$ be a left artin ring and let $P$ be an indecomposable projective $\Gamma$-module of Loewy length $n+1$. Then there is a natural map of $\operatorname{rings} \psi: \operatorname{End}_{\Gamma}(P / r P) \rightarrow \operatorname{End}_{\Gamma}\left(r^{n} P\right)$. A description of $\psi$ can be given as follows. Given $\alpha \in \operatorname{End}_{\Gamma}(P / r P)$, we can find an isomorphism $\beta: P \rightarrow P$ such that $\beta$ induces $\alpha$. Then $\psi(\alpha)$ is the restriction of $\beta$ to $r^{n} P$. One easily shows that $\psi(\alpha)$ is independent of the choice of $\beta$ and that $\psi$ is a ring map. Note that $\psi$ is always a monomorphism.

We now prove the main result of this section.

THEOREM 5.1. Let $\Gamma$ be a basic artin ring of Loewy length $n+1$ with radical $r$. Let $\Lambda=\Gamma / r^{n}$. Let $P_{1}, \ldots, P_{m}$ be a full set of non-isomorphic indecomposable left projective $\Gamma$-modules. Order the $P_{i}$ so that $L L\left(P_{i}\right)=n+1$ for $1 \leqq i \leqq s$ and $L L\left(P_{i}\right)<n+1$ for $s+1 \leqq i \leqq m$. Then $\Gamma$ is a selfinjective ring if and only if the following two conditions hold.

(1) $r P_{1}, \ldots, r P_{s}, P_{s+1}, \ldots, P_{m}$ is a full set of indecomposable injective $\Lambda$-modules.

(2) The induced ring maps $\operatorname{End}_{\Gamma}\left(P_{i} / r P_{i}\right) \rightarrow \operatorname{End}_{\Gamma}\left(r^{n} P_{i}\right)$ are isomorphisms for $i=1, \ldots, s$. 
Proof. Suppose that $\Gamma$ is selfinjective. Then since $\Gamma$ is basic, (1) immediately holds. Since each $P_{i}$ is injective and, for $1 \leqq i \leqq s, \operatorname{soc}\left(P_{i}\right)=r^{n} P_{i},(2)$ also follows.

Now suppose (1) and (2) hold. Under these hypotheses we begin by proving a lemma.

LeMma 5.2. Let $P, P^{\prime}$ be indecomposable projective $\Gamma$-modules with $L L(P)=n+1$. Then every map $f: r P \rightarrow P^{\prime}$ can be extended to a map $f^{*}: P \rightarrow P^{\prime}$.

Proof. First suppose that $L L\left(P^{\prime}\right) \leqq n$. Then $f$ is not a monomorphism since $\operatorname{soc}(P) \neq$ $\operatorname{soc}\left(P^{\prime}\right)$ and both socles are simple. Thus $f: r P \rightarrow P^{\prime}$ factors into $r P \rightarrow r P / r^{n} P \rightarrow P^{\prime}$. By the $\Lambda$-injectivity of $P^{\prime}$ we get the following commutative diagram which gives the desired result.

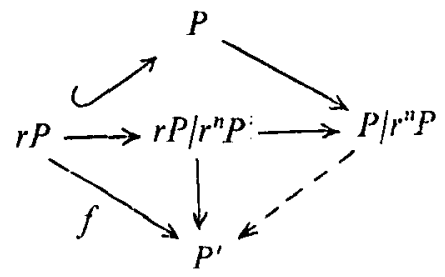

Thus we are reduced to considering the case in which $L L(P)=L L\left(P^{\prime}\right)=n+1$. If $f$ is not a monomorphism, then a similar argument to the one given above works, replacing $P^{\prime}$ by $r P^{\prime}$ and noting that $f(r P) \subseteq r P^{\prime}$.

Now suppose that $f$ is a monomorphism. Then $r^{n} P \cong r^{\prime \prime} P^{\prime}$, which implies that $P \cong P^{\prime}$, by (1). By (2), there exists $g: P \rightarrow P^{\prime}$ such that $g\left|r^{n} P=f\right| r^{n} P$, where $g \mid r^{n} P$ and $f \mid r^{n} P$ denote the maps restricted to $r^{n} P$. Then $(f-g) \mid r P: r P \rightarrow r P^{\prime}$ is not a monomorphism. Hence by above work, there exists $h: P \rightarrow P^{\prime}$ such that $h|r P=(f-g)| r P$. Then $g+h$ extends $f$.

We now return to the proof of the theorem. Let $P$ be an indecomposable $\Gamma$-projective. We want to show that $P$ is an injective module. Let $I$ be a left ideal in $\Gamma$ and let $f: I \rightarrow P$. We will show that there exists $f^{*}: \Gamma \rightarrow P$ such that

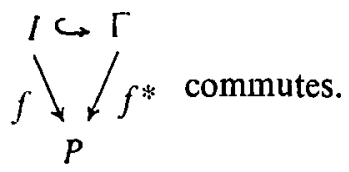

Case 1: $f$ is not a surjection. Then $f(I) \subset r P$ and hence $r^{n} I \subset \operatorname{ker} f$. Hence we have the following commutative diagram:

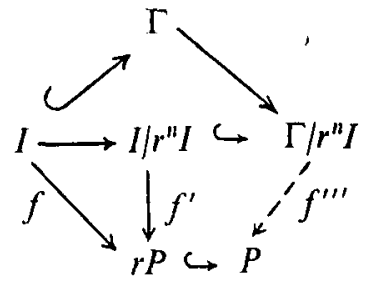

Thus it suffices to show that we can find $f^{\prime \prime \prime}: \Gamma / r^{n} I \rightarrow P$ making the diagram commutative.

Now $r^{n} I=\coprod_{j \in J_{1}} r^{n} P_{j}$, where $J_{1}$ is a subset of $\{1, \ldots, s\}$. Let $J_{2}=\{1, \ldots, s\}-J_{1}$. Then 


$$
\begin{gathered}
\Gamma / r^{n} I \cong \coprod_{j \in J_{1}} P_{j} / r^{n} P_{j} \coprod_{j \in J_{2}} P_{j} \coprod_{j=s+1}^{m} P_{j} . \text { Since } L L\left(I / r^{n} I\right) \leqq n, I / r^{n} I \rightarrow \Gamma / r^{n} I \text { factors into } \\
I / r^{n} I \rightarrow \coprod_{j \in J_{1}} P_{j} / r^{n} P_{j} \coprod_{j \in J_{2}} r P_{j} \coprod_{j=s+1}^{m} P_{j} \rightarrow \Gamma / r^{n} I .
\end{gathered}
$$

Since either $r P$ or $P$ is an injective $\Gamma / r^{n}$-module, depending on the Loewy length of $P, f^{\prime}: I / r^{n} I \rightarrow r P$ extends to

$$
f^{\prime \prime}: \coprod_{j \in J_{1}} P_{j} / r^{n} P_{j} \coprod_{j \in J_{2}} r P_{j} \prod_{j=s+1}^{m} P_{j} \rightarrow P .
$$

Finally, by Lemma $5.2, f^{\prime \prime}$ extends to a $\operatorname{map} f^{\prime \prime \prime}: \Gamma / r^{n} I \rightarrow P$.

Case 2: $f: I \rightarrow P$ is a surjection. Then since $P$ is projective, there exists $g: P \rightarrow I$ such that $f g=1_{P}$. Let $i: I \rightarrow \Gamma$ be the inclusion map and $\pi: \Gamma \rightarrow P$ be the canonical projection.

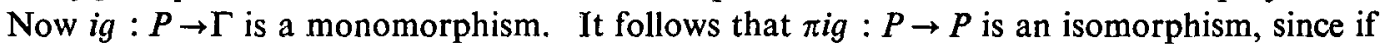
not, $\operatorname{soc}(P) \subset \operatorname{ker} \pi^{\prime} i g$ for all projections $\pi^{\prime}: \Gamma \rightarrow P^{\prime}$ and hence $\operatorname{soc}(P) \subset \operatorname{ker} i g=(0)$; contradiction. Hence there is a splitting $h: \Gamma \rightarrow P$ such that hig $=1_{p}$. Now consider $f-h i: I \rightarrow P$. Then $(f-h i) g=0$. Since $I$ decomposes into $I=I^{\prime} \amalg g(P)$, we have that $f-h i: I \rightarrow P$ factors into $I \rightarrow I^{\prime} \rightarrow P$. Letting $S=\operatorname{soc}(P)$, then $S$ is not a summand of $\operatorname{soc}\left(I^{\prime}\right)$, by (1) and the fact that $\Gamma$ is basic. Thus any map $I^{\prime} \rightarrow P$ is not a surjection and so $f-h i: I \rightarrow P$ is not a surjection. Hence, by Case 1 , there is an $h^{\prime}$ such that $f-h i=h^{\prime} i$. Thus $f=\left(h+h^{\prime}\right) i$ and this completes the proof.

The preceding theorem enables us to deduce some necessary conditions on an artin ring $\Lambda$ of Loewy length $n \geqq 2$ for there to be a selfinjective ring $\Gamma$ of Loewy length $n+1$, with $\Gamma / r^{\prime n} \cong \Lambda$, where $r^{\prime}$ denotes the radical of $\Gamma$. It is clearly necessary that we have a pairing $\left(P_{i}, E_{l}\right)$ of indecomposable projective and injective $\Lambda$-modules satisfying the following properties:

(i) if $L L\left(P_{i}\right)<n$, then $P_{i} \cong E_{i}$;

(ii) if $L L\left(P_{i}\right)=n$, then either $P_{i} \cong E_{i}$ or there is an isomorphism $u_{i}: r P_{i} \rightarrow E_{i} / \operatorname{soc}\left(E_{i}\right)$;

(iii) $\operatorname{End}\left(P_{i} / r P_{i}\right)$ and $\operatorname{End}\left(\operatorname{soc}\left(E_{i}\right)\right)$ are isomorphic.

We do not know whether these conditions are sufficient for the existence of a selfinjective ring $\Gamma$ with the above properties.

If $L L(\Lambda)=2$ and $\Lambda=\Lambda / r \ltimes r$, we get necessary and sufficient conditions for the existence of a selfinjective ring $\Gamma$ by combining the discussion in this section with our results in the first two sections. We need some preliminary results.

We shall first discuss what it means for an artin ring $\Gamma$ that its (left) socle, soc $\Gamma$, is the sum of one copy of each of the simple $\Gamma$-modules. In the same way as in Section 2 , it is easy to see that if $\Gamma$ is an artin algebra with this property, then $\Gamma$ must be selfinjective. Certainly any selfinjective artin ring has this property.

Let $S$ and $S^{\prime}$ be simple $\Gamma$-modules. We say that $S$ is linked to $S^{\prime}$ if $S^{\prime}=\operatorname{soc} P_{S}$, where $P_{S}$ denotes the projective cover of $S$. We write $S \searrow S^{\prime}$ if $S$ is linked to $S^{\prime}$.

LEMMA 5.3. Let $\Gamma$ be an artin ring with radical $r$ such that $\operatorname{soc}(\Gamma)$ is isomorphic to $\Gamma / r$, as left $\Gamma / r$-modules. If $S_{1}$ is a simple $\Gamma$-module, there exists a unique sequence of simple $\Gamma$-modules, $S_{1}, S_{2}, \ldots, S_{n}$ such that $S_{1} \searrow S_{2} \searrow \ldots \searrow S_{n} \searrow S_{1}$. 
Proof. By the hypothesis, if $S$ is a simple $\Gamma$-module then soc $P_{S}$ is simple. Using the fact that $\Gamma$ is basic, the result follows easily.

PROPOSITION 5.4. Let $\Gamma$ be an artin ring with radical $r$ such that $\operatorname{soc}(\Gamma)$ is isomorphic to $\Gamma / r$ as left $\Gamma / r$-modules. If $L L(\Gamma)=3$, then $\Gamma$ decomposes as a ring into a product of artin rings $\Gamma_{1} \times \Gamma_{2} \times \Gamma_{3}$ such that $L L\left(\Gamma_{i}\right)=i$ and the left indecomposable projective $\Gamma_{i}$-modules all have Loewy length $i$.

Proof. Let $P$ be an indecomposable projective of Loewy length 3 . Let $S=P / r P$ and $T=r^{2} P$. Let $P^{\prime}$ be the projective cover of $T$. We first show that $L L\left(P^{\prime}\right)=3 . \quad L L\left(P^{\prime}\right)$ cannot be 1 since $\operatorname{soc}\left(P^{\prime}\right) \neq \operatorname{soc}(P)$. If $L L\left(P^{\prime}\right)=2$, choose a simple $S^{\prime \prime} \hookrightarrow P / r^{2} P$ and let $P^{\prime \prime}$ be the projective cover of $S^{\prime \prime}$. If $S^{\prime \prime}$ was projective, $S^{\prime \prime}$ would be a summand of $r P$, which is impossible since $r P$ has simple socle. Hence we conclude that $L L\left(P^{\prime \prime}\right) \neq 1 . \quad L L\left(P^{\prime \prime}\right) \neq 2$ since if so $\operatorname{soc}\left(P^{\prime \prime}\right)=T=\operatorname{soc}(P)$, a contradiction. Thus $L L\left(P^{\prime \prime}\right)=3$. Now $T \hookrightarrow P^{\prime \prime} / r^{2} P^{\prime \prime}$ and hence the induced map $P^{\prime} \rightarrow P^{\prime \prime}$ is a monomorphism since $L L\left(P^{\prime}\right)=2$ and $\operatorname{soc}\left(P^{\prime}\right)$ is simple. Thus $\operatorname{soc}\left(P^{\prime}\right) \cong \operatorname{soc}\left(P^{\prime \prime}\right)$, a contradiction. Hence we have shown that $L L\left(P^{\prime}\right)=3$. By the hypothesis it is easy to see that indecomposable projectives of Loewy length 1 are also injective. Thus one easily sees that $\Gamma=\Gamma_{1} \times \Gamma^{\prime}$ where $\Gamma_{1}$ is a semi-simple ring and $\Gamma^{\prime}$ has no simple projectives. Let $P$ and $P^{\prime}$ be indecomposable projectives with $L L(P)=2$ and $L L\left(P^{\prime}\right)=3$. It remains to show that $\operatorname{Hom}\left(P, P^{\prime}\right)=0$ and $\operatorname{Hom}\left(P^{\prime}, P\right)=0$. By the first part of the proof, $\operatorname{Hom}\left(P, P^{\prime}\right)=(0)$. Now let $f \in \operatorname{Hom}\left(P^{\prime}, P\right)$. It follows that if $f \neq 0$, then $f\left(P^{\prime}\right)=r P=\operatorname{soc}(P)$. Thus $P^{\prime} / r P^{\prime} \cong$ $\operatorname{soc}(P)$. Let $T_{1}=P^{\prime} / r P^{\prime}$ and let $T_{1} \searrow T_{2} \searrow \ldots \searrow T_{n} \searrow T_{1}$ be the unique sequence of simple modules (see Lemma 5.3). Then by the first part of the proof, since $L L\left(P^{\prime}\right)=3$, the Loewy lengths of the projective covers of each of the $T_{i}$ 's is 3 . Let $P_{n}$ be the projective cover of $T_{n}$. Then $L L\left(P_{n}\right)=3$ and $\operatorname{soc}\left(P_{n}\right)=T_{1}=\operatorname{soc}(P)$. Since $L L(P)=2$, this is a contradiction and this completes the proof.

This proposition shows that if $\Lambda$ is an artin ring with $r^{2}=0$ and there exists a selfinjective artin ring $\Gamma$ such that $r^{\prime 3}=0$ and $0 \rightarrow r^{\prime 2} \rightarrow \Gamma \rightarrow \Lambda \rightarrow 0$ is exact, where $r^{\prime}$ is the radical of $\Gamma$, then $\Lambda$ decomposes into a product $\Lambda_{1} \times \Lambda_{2} \times \Lambda_{3}$ corresponding to the decomposition of $\Gamma$. It is worth noting that one can find an example of an indecomposable selfinjective basic artin ring $\Gamma$ of Loewy length 4 with indecomposable projectives of Loewy lengths 3 and 4 . We are now in a position to state the result for $\Lambda=\Lambda / r \times r$ and $L L(\Lambda)=2$.

PROPOSITION 5.5. Let $\Lambda=\Lambda / r \propto r$ be an indecomposable artin ring with $L L(\Lambda)=2$. Then there is some selfinjective ring $\Gamma$ with $L L(\Gamma)=3$ such that $\Gamma / r^{\prime 2} \cong \Lambda$ if and only if we have triples $\left(P_{i}, E_{i}, u_{i}\right)$, where the $P_{i}$ are the indecomposable projective $\Lambda$-modules, the $E_{i}$ the indecomposable injective $\Lambda$-modules and the $u_{i}: r P_{l} \rightarrow E_{i} / r E_{i}$ isomorphisms, such that condition $A$ is satisfied, and the natural map from $\operatorname{End}\left(P_{i}\right)$ to End( $\left.\operatorname{soc} E_{i}\right)$, obtained by sending $\alpha$ to $\gamma$ in the notation of Section 1, is an isomorphism.

\section{REFERENCES}

1. M. Auslander, Representation dimension of Artin algebras (Queen Mary College Notes, 1971).

2. M. Auslander and I. Reiten, Notes on the representation theory of Artin algebras (Brandeis Univ., 1972). 
3. H. Cartan and S. Eilenberg, Homological algebra (Princeton University Press, 1956).

4. S. Eilenberg, H. Nagao and T. Nakayama, On the dimension of modules and algebras IV: Dimension of residue rings of hereditary rings, Nagoya Math. J. 10 (1956), 87-95.

5. R. Fossum, P. Griffith and I. Reiten, Trivial extensions of abelian categories, Lecture Notes in Mathematics No. 456 (Springer-Verlag, 1975).

6. P. Gabriel, Indecomposable representations I, Manuscr. Math. 6 (1972), 71-103.

7. E. Green and W. Gustafsen, Pathological Quasi-Frobenius algebras of finite type, Communications in algebra 2 (1974), 233-260.

Department of Mathematics

University of Pennsylvania

PhILAdELPHIA 19104
DePaRTMENT OF MATHEMATICS

UNIVERSITY OF TRONDHEIM

7000 TRONDHEIM

NORWAY 